\title{
Cuidados paliativos no contexto da pandemia de COVID-19: desafios e contribuições
}

Palliative care amidst the COVID-19 pandemic: challenges and contributions Cuidados paliativos en el contexto de la pandemia de COVID-19: desafíos y contribuciones

Raquel Sampaio Florêncio ${ }^{1}$ id https://orcid.org/0000-0003-3119-7187

Virna Ribeiro Feitosa Cestari ${ }^{1}$ ic https://orcid.org/0000-0002-7955-0894

Lorena Campos de Souza ${ }^{1}$ id https://orcid.org/0000-0002-3338-9425

Amanda Caboclo Flor ${ }^{1}$ io https://orcid.org/0000-0002-4698-0126

Vitória Pessoa Nogueira ${ }^{1}$ io https://orcid.org/0000-0002-6150-8938

Thereza Maria Magalhães Moreira1 io https://orcid.org/0000-0003-1424-0649

Marina de Góes Salvetti ${ }^{2}$ io https://orcid.org/0000-0002-4274-8709

Vera Lúcia Mendes de Paula Pess0a ${ }^{1}$ io https://orcid.org/0000-0002-5441-5311

Florêncio RS, Cestari VR, Somo citar: Nogueira VP, Moreira TM, et al. Cuidados paliativos no contexto da pandemia de COVID-19: desafios e contribuições. Acta Paul Enferm. 2020;33:eAPE20200188.

DOI http://dx.doi.org/10.37689/actaape/2020A001886

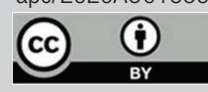
Cuidados paliativos; Coronavírus; Infecções por coronavirus; COVID-19; Pandemias; COVID-19

Keywords Palliative care; Coronavírus; Coronavirus infections; COVID-19; Pandemics

Descriptores

Cuidados paliativos; Coronavírus; Infecciones por coronavirus; COVID-19; Pandemias

\section{Submetido \\ 20 de Julho de 2020 \\ Aceito \\ 23 de Setembro de 2020}

Autor correspondente

Raquel Sampaio Florêncio

E-mail: raquelsampy@hotmail.com

\section{Resumo}

Objetivo: Analisar as evidências científicas sobre a inserção dos cuidados paliativos no cenário da pandemia de COVID-19.

Método: Revisão de escopo baseada na metodologia proposta pelo Instituto Joanna Briggs. Foram analisadas as bases da Biblioteca Virtual da Saúde, Web of Science, Scopus, Cumulative Index to Nursing and Allied Health Literature, EBSCO, Embase, Psyclnfo® e Science Direct, por meio dos descritores "cuidados paliativos" e "coronavirus" e a palavra-chave "COVID-19". Foram selecionados 30 estudos para análise.

Resultados: A revisão evidenciou estratégias para implementação dos cuidados paliativos com a utilização de protocolos e telemedicina, para facilitar a comunicação, e mostrou como tem sido a abordagem da terapêutica paliativista, revelando a necessidade da promoção do conforto e do acolhimento de pacientes e familiares em momentos de dor e luto.

Conclusão: Os princípios dos cuidados paliativos contribuem para o cuidado integral, mas sua implementação no contexto de crise é um desafio. É importante proporcionar condições para aplicá-lo no cotidiano dos serviços de saúde, mesmo em cenários de pandemia.

\section{Abstract}

Objective: To analyze the scientific evidence on palliative care implementation amidst the COVID-19 pandemic. Method: This is a scoping review based on the methodology proposed by the Joanna Briggs Institute. Virtual Health Library, Web of Science, Scopus, Cumulative Index to Nursing and Allied Health Literature, EBSCO, Embase, Psyclnfo ${ }^{\circledR}$ and Science Direct were analyzed using the descriptors "palliative care" and "coronavirus" and the keyword "COVID-19". Thirty studies were selected for analysis.

Results: This review showed strategies for palliative care implementation using protocols and telemedicine, to facilitate communication, and showed how palliative therapy approach has been, revealing the need to promote comfort and welcoming patients and family members in times of pain and grief.

Conclusion: The palliative care principles contribute to comprehensive care, but its implementation amidst the crisis is a challenge. It is important to provide conditions to apply it in the daily life of health services, even in pandemic scenarios.

\section{Resumen}

Objetivo: Analizar las evidencias científicas sobre la incorporación de los cuidados paliativos en el escenario de la pandemia de COVID-19. 
Métodos: Revisión de alcance basada en la metodología propuesta por el Instituto Joanna Briggs. Se analizaron las bases de la Biblioteca Virtual da Saúde, Web of Science, Scopus, Cumulative Index to Nursing and Allied Health Literature, EBSCO, Embase, Psyclnfo® y Science Direct, mediante los descriptores "cuidados paliativos" y "coronavirus" y la palabra clave "COVID-19". Se seleccionaron 30 estudios para el análisis.

Resultados: La revisión evidenció estrategias para la implementación de los cuidados paliativos con el uso de protocolos y telemedicina para facilitar la comunicación y demostró de qué forma se está realizando el enfoque de la terapia paliativa, que reveló la necesidad de promover el bienestar de pacientes y familiares y acogerlos en momentos de dolor y duelo.

Conclusión: Los principios de los cuidados paliativos contribuyen para el cuidado integral, pero su implementación en el contexto de crisis es un desafío. Es importante proporcionar condiciones para aplicarlo en la cotidianidad de los servicios de salud, inclusive en escenarios de pandemia.

\section{Introdução}

A condição de pandemia determinada pela Organizaçáo Mundial de Saúde (OMS) em março de 2020 repercutiu de forma intensa na área da saúde, expondo limitaçóes importantes em termos de insumos e profissionais da saúde capacitados para lidarem com a doença pelo coronavírus 2019 (COVID-19). A despeito do perfil epidemiológico delineado inicialmente, além de indivíduos com idades superiores a 70 anos e aqueles com comorbidades, jovens e crianças também passaram a ser acometidos. ${ }^{(1-3)}$

Em todo o mundo, o volume de casos confirmados aumenta de modo exponencial, o que resulta em estresse para os serviços de saúde, além de importante desequilíbrio entre a oferta e a procura de leitos de terapia intensiva. Tal panorama traz à tona discussóes sobre um tópico de difícil assimilação por parte da sociedade: a quem assegurar assistência e recursos tecnológicos para recuperação do quadro clínico imposto pelo novo coronavírus?

Ao considerarmos o direito à saúde como condição extensiva a todos os cidadãos, resulta em impotência e angústia a necessidade da tomada de decisão em circunstâncias tão adversas. Sob tal cenário, os fundamentos que norteiam os cuidados paliativos podem ser utilizados como imperativo ético na otimização de recursos.

Segundo a OMS, o cuidado paliativo é uma abordagem que melhora a qualidade de vida dos pacientes e suas famílias enfrentando os problemas associados à doença com risco de morte, pela prevenção e pelo alívio do sofrimento, por meio da identificação precoce, da avaliação impecável e do tratamento da dor e outros problemas físicos, psicossociais e espirituais. ${ }^{(4)}$ A relevância dessa prática levou a própria OMS a atualizar o documento Clinical management of COVID-19 e incluir um ca- pítulo acerca dos cuidado paliativo, recomendando um plano de cuidados para COVID-19 e respeito às preferências dos pacientes e de seus familiares. ${ }^{(5)}$

Situaçóes de pandemia, como a atual, demandam grande sobrecarga aos serviços de saúde, que poderiam se beneficiar a oferta de cuidados paliativos. ${ }^{(6)}$ Em contextos emergenciais e de crise humanitária, medidas para aliviar o sofrimento podem ser negligenciadas, em razão da necessidade imediata de salvar vidas. Contudo, vale ressaltar que os cuidados paliativos são igualmente primordiais, e sua ausência implica em medidas de enfrentamento falhas e eticamente insustentáveis, como o delineado pela COVID-19. ${ }^{(7)}$

Instituir os cuidados paliativos torna-se uma opção viável para assegurar cuidado de qualidade, enquanto se faz necessário decidir quem precisa ou não de cuidados intensivos para manutenção da vida. Por outro lado, diante do sem número de pessoas afetadas pela COVID-19 e da presença de sinais e sintomas complexos, os cuidados paliativos se apresentam como possibilidade de conforto e alívio do sofrimento diante de prognósticos incertos. ${ }^{\left({ }^{(}\right)}$

Ampliar a discussão em torno da inclusão dos cuidados paliativos nas estratégias de assistência aos pacientes que cursam com a COVID-19 faz-se necessário, não apenas pela premente exigência de otimização de leitos e recursos, mas também pelo direito de se ter assegurada a dignidade e o conforto diante de uma doença que ameaça a vida.

O objetivo deste estudo foi analisar as evidências científicas sobre a inserção dos cuidados paliativos no cenário da pandemia da COVID-19.

\section{Métodos}

Trata-se de uma revisão de escopo, uma forma de síntese de conhecimento que aborda uma questão 
de pesquisa exploratória, destinada a mapear os conceitos-chave (evidências) que apoiam determinada área de pesquisa, identificar lacunas nas evidências existentes e emergentes e estabelecer metas para pesquisas futuras. ${ }^{(9,10)}$ A construção desta revisão de escopo seguiu a estrutura de Peters et al., ${ }^{(1,12)}$ publicada pelo Instituto Joanna Briggs. ${ }^{(13)}$

Para identificação da questão da pesquisa, utilizou-se a estratégia População, Conceito e Contexto, na qual "P" corresponde à população/participantes (pacientes com COVID-19) e "C" ao conceito que se pretende investigar (cuidados paliativos). Optouse por não inserir o Contexto, pois buscou-se explorar as evidências da relação entre a COVID-19 e os cuidados paliativos. Assim, esta revisão considerou a seguinte questão de pesquisa: Como tem ocorrido a implementaçáo dos cuidados paliativos para os pacientes com COVID-19?

Para os propósitos da presente pesquisa, a fonte de informaçóes incluiu estudos publicados no ano de 2020, em qualquer idioma e que estivessem disponíveis eletronicamente na íntegra. Relatórios de pesquisa e resumos publicados em anais de eventos foram definidos como critérios de exclusão.

A busca pareada se deu nas bases de dados eletrônicas da Biblioteca Virtual da Saúde (BVS), ScienceDirect, Web of Science, Scopus, Cumulative Index to Nursing and Allied Health Literature (CINAHL), EBSCO, Embase e PsycInfo ${ }^{\circledR}$. Foram utilizados os descritores de acordo com a terminologia dos Descritores em Ciência da Saúde (DeCS) e Medical Subject Headings (MeSH). Reitera-se que houve a inclusão das palavras-chave "COVID-19" e "COVID 19" nas buscas, no intuito de ampliar os achados. Com esses termos, foram realizadas buscas, integrando-os mediante os operadores booleanos “ $A N D$ ” e "OR".

As estratégias de buscas para cada base estão descritas no quadro 1. Optou-se por realizar a busca com os descritores na língua inglesa, pois a equação possibilitou a identificação de maior quantidade de artigos, quando comparada às buscas com descritores nas línguas portuguesa e espanhola.

Os dados foram extraídos dos artigos incluídos na revisão de escopo por dois revisores independentes. Os artigos selecionados foram organizados em
Quadro 1. Estratégias de buscas utilizadas na Biblioteca Virtual da Saúde, Web of Science, Scopus, Cumulative Index to Nursing and Allied Health Literature, EBSCO, Embase, PsycInfo® e Science Direct

\begin{tabular}{|l|l|}
\hline Base & Estratégias de busca \\
\hline Biblioteca Virtual da Saúde & $\begin{array}{l}\text { (tw:(coronavírus or coronavirus or "covid-19" or "covid } \\
\text { 19")) AND (tw:("cuidados paliativos" or "cuidado paliativo" } \\
\text { or "palliative care")) }\end{array}$ \\
\hline Web of Science, Science Direct & $\begin{array}{l}\text { (coronavirus or "COVID-19" or "COVID 19") ANDTÓPICO: } \\
\text { ("palliative care") }\end{array}$ \\
\hline Scopus & $\begin{array}{l}\text { (TITLE-ABS-KEY (coronavirus OR "covid-19" OR "covid } \\
19 ") \text { AND TITLE-ABS-KEY ("palliative care")) }\end{array}$ \\
\hline EBSCO, CINAHL & $\begin{array}{l}\text { (coronavirus OR "covid-19" OR "covid 19") AND } \\
\text { ("palliative care") }\end{array}$ \\
\hline EMBASE & $\begin{array}{l}\text { ('coronavirus'/exp OR coronavirus OR 'covid-19' OR } \\
\text { 'covid 19'/exp OR 'covid 19') AND ("palliative care'/exp } \\
\text { OR 'palliative care') }\end{array}$ \\
\hline PSycInfo & $\begin{array}{l}\text { Any Field: coronavirus OR Any Field: "COVID-19" OR Any } \\
\text { Field: "COVID 19" AND Any Field: "palliative care" }\end{array}$ \\
\hline
\end{tabular}

CINAHL - Cumulative Index to Nursing and Allied Health Literature

um banco de dados do Microsoft Excel $^{\oplus}$ com descrição de sua identificação (título, autores, idioma, país, local, periódico e ano); aspectos metodológicos (delineamento de pesquisa, objetivos, amostragem, tratamento de dados e nível de evidência) e análise crítica (resultados e conclusóes).

Controvérsias foram solucionadas por meio de discussão crítica entre os revisores. $\mathrm{O}$ rascunho da ferramenta de extração de dados foi modificado e revisado conforme necessário durante o processo realizado para cada estudo incluído. As buscas e o processo de seleção dos estudos ocorreram durante o mês de abril de 2020.

Por se tratar de revisão sistemática de escopo, segundo a metodologia utilizada, é dispensada a avaliação de qualidade metodológica dos estudos incluídos. Para a elaboração da revisão, seguiram-se as recomendaçóes do checklist Preferred Reporting Items for Systematic Reviews and Meta-Analyses - extension for Scoping Review (PRISMA-ScR) ${ }^{(14)}$ e o fluxograma PRISMA. ${ }^{(15)}$

\section{Resultados}

A pesquisa identificou 170 estudos potencialmente relevantes, dos quais 25 foram removidos por serem duplicados. Foram analisados 145 resumos, sendo eliminados 104 publicados antes do ano de 2020 . Destes, 41 resumos foram avaliados quanto à elegibilidade e, ao final, restaram 30 artigos para análise 


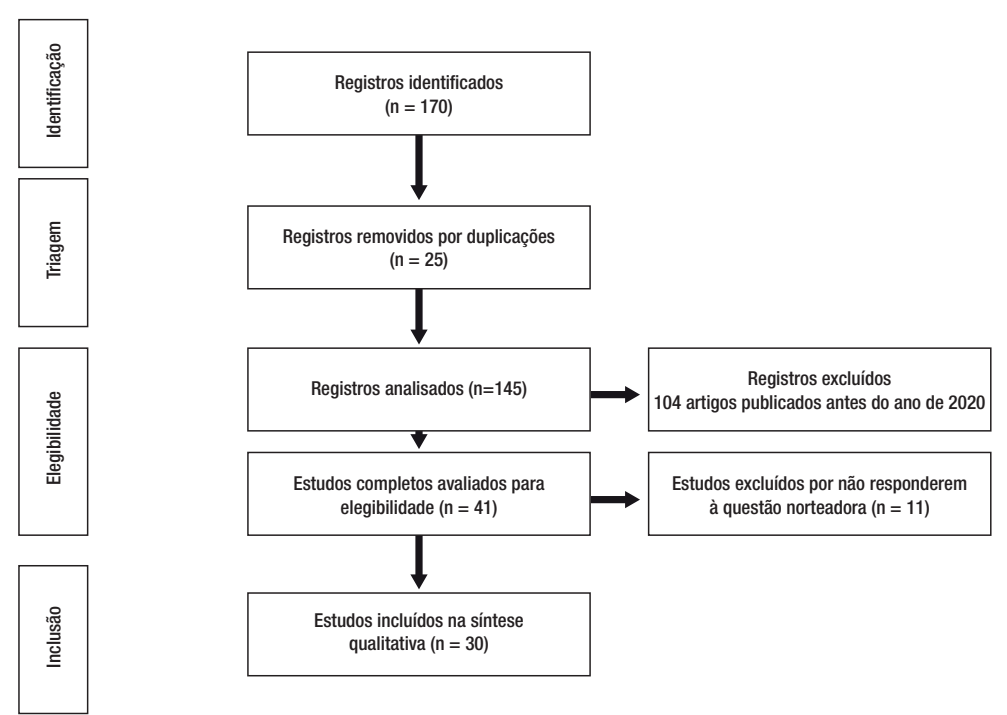

Figura 1. Diagrama do processo de inclusão e exclusão dos estudos

do texto na íntegra por dois revisores independentes (Figura 1).

As características dos estudos incluídos nesta revisão estão detalhadas no quadro 2. Eles foram desenvolvidos em nove países, com destaque para a produção dos Estados Unidos (11; 36,6\%) e Reino Unido (8; 26,7\%). Canadá, Alemanha, Suíça e Itália publicaram dois artigos cada (6,7\%), e Nigéria, China e Nova Zelândia, um artigo cada (3,3\%). Os periódicos Journal of Pain and Symptom Management e Royal College of Physicians foram os que mais publicaram sobre a temática, com 12 (40\%) e dois (6,7\%) artigos, respectivamente. Os demais periódicos tiveram apenas uma $(3,3 \%)$ publicação cada.

No tocante ao tipo de estudo, sete $(23,3 \%)$ eram recomendaçôes de especialistas; seis (20\%) eram relatos de experiência; cinco (16,7\%) revisóes da literatura não especificadas; quatro $(13,3 \%)$ editoriais; dois $(6,7 \%)$ estudos transversais e descritivos. Os demais foram artigos de atualização, de opiniāo, revisão sistemática, relatos de caso e reflexivos. A fonte dos dados dos estudos foi em sua maioria, da literatura científica $(16 ; 53,3 \%)$, seguida das vivências dos autores (6; $20 \%)$, de pesquisa de campo $(3 ; 10 \%)$ e de contato via telefone $(1 ; 3,3 \%)$. Nos editoriais, a fonte foi a literatura e vivências $(4 ; 13,3 \%)$.
Em 16 estudos, observou-se a preocupação dos cuidados paliativos em diferentes populaçóes, como crianças $(1 ; 6,2 \%)$; adultos e idosos (6; $37,5 \%)$; pacientes com câncer $(1 ; 6,2 \%)$; população LGBTQ+ (1; 6,2\%); profissionais gerais da saúde $(3 ; 18,7 \%)$; equipes de cuidados paliativos ( $2 ; 12,5 \%)$, e um artigo $(6,2 \%)$ abordou tanto pacientes, como os demais prestadores de cuidados e profissionais da saúde.

Em relação às temáticas, duas categorias sintetizam os principais aspectos abordados nos estudos: "Estratégias para implementação dos cuidados paliativos no enfrentamento da COVID-19" (17-19,21,23,25,27-32,35,36,38-42,44)(19; 63,3\%) e "Terapêutica paliativista no contexto da pandemia: desafios e contribuiçóes”"(16,19-29,31,33,34,36-41,43-45) (24;80\%).

Entre as estratégias utilizadas para implementaçáo dos cuidados paliativos no enfrentamento da COVID-19, destacaram-se a telemedicina e a comunicaçáo entre os atores envolvidos, ${ }^{(17-19,21,25,27,28,30,32,35,36,38-42,44)}$ o planejamento das açôes, ${ }^{(17-19,21,23,25,32,35,39-41,44)}$ a utilização adequada dos equipamentos de proteção individual e outros materiais ${ }^{(19,21,27,28,36,39)}$ e o uso de ferramentas de gestáo como planos, diretrizes e protocolos. ${ }^{(18,21,23,25,27,28,30-32,35,39,40,42,44)}$ 
Quadro 2. Caracterização dos artigos selecionados para esta revisão de escopo

\begin{tabular}{|c|c|c|c|}
\hline Autoria & Tipo de estudo & População/amostra & $\begin{array}{l}\text { Fonte dos } \\
\text { dados }\end{array}$ \\
\hline Weaver et al. ${ }^{(16)}$ & \begin{tabular}{|l} 
Recomendações de \\
especialistas
\end{tabular} & Crianças & Literatura \\
\hline Calton et al. ${ }^{(17)}$ & \begin{tabular}{|l} 
Recomendações de \\
especialistas
\end{tabular} & $\begin{array}{l}\text { Pacientes em cuidados } \\
\text { paliativos }\end{array}$ & Literatura \\
\hline Powell et al. ${ }^{(18)}$ & \begin{tabular}{|l|}
$\begin{array}{l}\text { Recomendaç̃os de } \\
\text { especialistas }\end{array}$ \\
\end{tabular} & $\begin{array}{l}\text { Equipe de cuidados } \\
\text { paliativos }\end{array}$ & Literatura \\
\hline Hendin et al. ${ }^{(19)}$ & $\begin{array}{l}\text { Recomendações de } \\
\text { especialistas }\end{array}$ & $\begin{array}{l}\text { Pacientes em cuidados } \\
\text { paliativos }\end{array}$ & Literatura \\
\hline Nehls et al. (20) & $\begin{array}{l}\text { Recomendação de } \\
\text { especialistas }\end{array}$ & - & Literatura \\
\hline Fausto et al. ${ }^{(21)}$ & Relato de experiência & Profissionais da saúde & Vivência \\
\hline Ferguson et al. ${ }^{(22)}$ & Atualização & Profissionais da saúde & Literatura \\
\hline Borasio et al. (23) & Relato de experiência & $\begin{array}{l}\text { Equipe de cuidados } \\
\text { paliativos }\end{array}$ & Vivência \\
\hline Rolanda et al. ${ }^{(24)}$ & Relato de experiência & $\begin{array}{l}\text { Idosos em casas de } \\
\text { repouso }\end{array}$ & Vivência \\
\hline Curtis et al. (25) & Artigo de opinião & Profissionais da saúde & Literatura \\
\hline The Lancet(26) & Editorial & - & $\begin{array}{l}\text { Literatura e } \\
\text { vivências }\end{array}$ \\
\hline Bajwah et al. ${ }^{(2 \pi)}$ & Revisão de literatura & - & Literatura \\
\hline Salako et al.(28) & Relato de experiência & Pacientes com câncer & Vivência \\
\hline $\operatorname{Fan}^{(29)}$ & Relato de experiência & $\mathrm{Nl}$ & Vivência \\
\hline Etkind et al. ${ }^{(30)}$ & Revisão sistemática & $\begin{array}{l}\text { Pacientes, prestadores de } \\
\text { cuidados e profissionais } \\
\text { da saúde }\end{array}$ & Literatura \\
\hline $\begin{array}{l}\text { Fusi-Schmidhauser } \\
\text { et al. }{ }^{(31)}\end{array}$ & Descritivo & $\begin{array}{l}\text { Pacientes com COVID-19 } \\
\text { não adequados para } \\
\text { ventilação }\end{array}$ & $\begin{array}{l}\text { Pesquisa de } \\
\text { campo }\end{array}$ \\
\hline Tran et al. ${ }^{(32)}$ & $\begin{array}{l}\text { Recomendações de } \\
\text { especialistas }\end{array}$ & - & Literatura \\
\hline Lovell et al..$^{(33)}$ & Transversal & $\begin{array}{l}101 \text { pacientes com } \\
\text { COVID-19 }\end{array}$ & $\begin{array}{l}\text { Pesquisa de } \\
\text { campo }\end{array}$ \\
\hline Rosa et al..$^{(34)}$ & \begin{tabular}{|l|} 
Recomendações de \\
especialistas
\end{tabular} & LGBTQ+ com COVID-19 & Literatura \\
\hline Humphreys et al. ${ }^{(35)}$ & Relato de experiência & $\mathrm{Nl}$ & Vivência \\
\hline Constantini et al.(36) & Transversal & $\begin{array}{l}16 \text { instituições de saúde } \\
\text { italianas }\end{array}$ & $\begin{array}{l}\text { Contato via } \\
\text { telefone }\end{array}$ \\
\hline Davies et al. ${ }^{(37)}$ & Revisão de literatura & - & Literatura \\
\hline Sun et al. ${ }^{(38)}$ & Relato de casos & $\begin{array}{l}30 \text { pacientes com COVID } \\
\text { em admitidos em unidade } \\
\text { de cuidados paliativos }\end{array}$ & $\begin{array}{l}\text { Pesquisa de } \\
\text { campo }\end{array}$ \\
\hline Romanò (39) & Revisão de literatura & - & Literatura \\
\hline Radbruch et al..$^{(40)}$ & Editorial & - & $\begin{array}{l}\text { Literatura e } \\
\text { vivências }\end{array}$ \\
\hline Feder et al. ${ }^{(41)}$ & Editorial & - & $\begin{array}{l}\text { Literatura e } \\
\text { vivências }\end{array}$ \\
\hline Chidiac et al. ${ }^{(42)}$ & Editorial & - & $\begin{array}{l}\text { Literatura e } \\
\text { vivências }\end{array}$ \\
\hline Peatel ${ }^{(43)}$ & Editorial & - & $\begin{array}{l}\text { Literatura e } \\
\text { vivências }\end{array}$ \\
\hline Arya et al. ${ }^{(44)}$ & Revisão de literatura & $\mathrm{Nl}$ & Literatura \\
\hline Wallace et al. ${ }^{(45)}$ & Reflexivo & - & Literatura \\
\hline
\end{tabular}

NI - não informado; LGBTQ+ - Lésbicas, Gays, Bissexuais, Travestis, Transexuais, Transgêneros e Queer

Já em relação aos desafios e contribuiçôes da terapêutica paliativista no contexto da pandemia, verificaram-se, principalmente, o manejo farmacológico e não farmacológico dos sinais e sintomas dos pacientes ${ }^{(19-22,24,25,27,29,31,33,36-39,44)}$ e o apoio emocional e espiritual aos pacientes, familiares e/

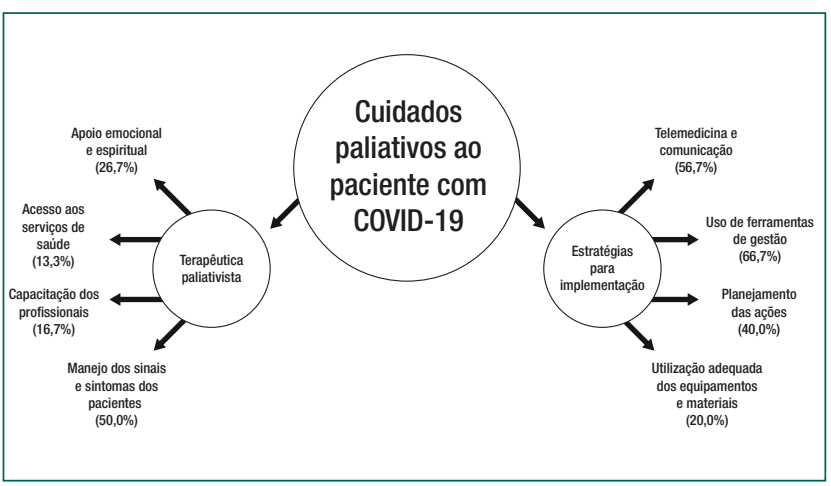

Figura 2. Categorias e subcategorias identificadas nos estudos

ou cuidadores. ${ }^{(23,26,34,40,41,43-45)}$ Os desafios estiveram relacionados ao acesso dos pacientes aos serviços de saúde, ${ }^{(26,34,36,44)}$ às necessidade de capacitação dos profissionais, ${ }^{(26,27,29,33,36)}$ à sobrecarga de trabalho e à falta de recursos, como retratado na figura $2 .{ }^{(26,36)}$

Alguns estudos estão tanto na primeira quanto na segunda categoria, pois apresentaram informaçóes pertinentes para ambas.

\section{Discussão}

Dentre as limitações, evidenciamos a falta de estudos prospectivos, que se justifica pelo caráter agudizado da doença e o aparecimento recente do vírus. Também é possível verificar que muitos artigos estavam baseados em experiências em algumas instituiçóes, que podem não se aplicar à realidade de outros países. Ademais, a sobrecarga dos profissionais da saúde e a busca urgente pela cura reforçam o caráter biomédico da atenção à saúde, em oposição aos cuidados paliativos, que enfatizam a humanização, o conforto e a qualidade de vida dos pacientes.

No cenário pandêmico, os cuidados paliativos se revelam diferentes, potentes e em múltiplas formas, com estudos e recomendaçóes de especialistas mostrando variadas possibilidades neste campo de cuidado e pesquisa, passando desde os princípios dos cuidados paliativos até os desafios e fragilidades em meio às consequências da COVID-19.

\section{Estratégias para implementação dos cuidados paliativos no enfrentamento da COVID-19}

A crescente demanda por cuidados paliativos devido à COVID-19 torna necessário o estabelecimen- 
to de estratégias para prestar tais cuidados, com a determinação de um modelo eficiente para que os serviços minimizem riscos e ampliem o alcance da abordagem paliativa. O objetivo é fornecer recomendaçóes aos profissionais de saúde sobre os cuidados paliativos; evitar tratamento intensivo oneroso, assegurando cuidados de qualidade a quem precisa; estruturar serviços capazes de prover atendimento rápido e eficaz e identificar adequadamente quem pode ou não se beneficiar de um tratamento intensivo invasivo. ${ }^{(21,23,30)}$

Para a efetivação dessa organização, é importante implementar protocolos para identificar pacientes que não se beneficiam de procedimentos extremos. (25) Ressalta-se que, para a efetividade dessa triagem, os serviços precisam contar com pelo menos uma equipe de cuidados paliativos como suporte para as equipes generalistas. Ademais, os cuidados paliativos durante a pandemia precisam se adaptar a um estilo emergencial, pois os pacientes podem se deteriorar rapidamente e exigir decisóes imediatas e planos de tratamento claros. Desse modo, os cuidados paliativos devem estar na vanguarda para ajudar a tomar as melhores decisóes, cuidar das famílias e oferecer apoio espiritual. ${ }^{(31)}$

A abordagem paliativa foi evidenciada no contexto da pandemia náo somente pela necessidade de estabelecer protocolos para os pacientes acometidos pela COVID-19, mas para reorganizar aspectos referentes aos demais pacientes, como a assistência àqueles em tratamento oncológico, ${ }^{(28)}$ para os quais a equipe multidisciplinar deve criar, revisar e atualizar rapidamente uma política clínica de combate a uma infecção pela COVID-19 durante o tratamento.

Os serviços de saúde devem contar com ofertas de medicaçóes de conforto, ter uma equipe multidisciplinar especializada, otimizar uso de espaço nos hospitais/clínicas de cuidados paliativos, adotar um sistema de triagem, prezar pela relação interpessoal, utilizar a telemedicina e fazer uso de protocolos, sendo fundamental uma abordagem multifacetada. ${ }^{(44)}$

No contexto de isolamento social, a equipe deve disponibilizar chamadas de vídeos para os pacientes entrarem em contato com seus familiares, para a equipe se comunicar com a família e até mesmo para capacitaçóes dos profissionais ou cuidadores.
Além de favorecer a comunicação, a telemedicina é fundamental diante da limitação do uso de Equipamentos de Proteção Individual, pois pode favorecer a proteção da equipe e a garantia de cuidados adequados aos pacientes internados com COVID-19. ${ }^{(18,28,30,35,44)}$ Para tanto, é necessário o acesso equitativo à tecnologia, levando em consideração as vulnerabilidades e as limitaçóes de pacientes e cuidadores, ${ }^{(17)}$ com vistas à promoçáo do conforto e do bem-estar. ${ }^{(19,27,38)}$

Em resposta a essa realidade, os profissionais da linha de frente devem receber treinamento para realizar os cuidados paliativos primários aos pacientes em condição grave, acionando a equipe de especialistas nesse tipo de cuidado sempre que necessário. ${ }^{(18,30)}$

A organização dos serviços de saúde, considerando os cuidados paliativos como eixo transversal a todas as áreas, é essencial no enfrentamento da pandemia causada pelo coronavírus da síndrome respiratória aguda grave 2 (SARS-CoV-2), pois há necessidade de equilibrar ética, conforto e assistência de qualidade. As equipes paliativistas são fundamentais, desde a triagem dos tratamentos até a necessidade de fornecer notícias e dar suporte aos familiares.

\section{Terapêutica paliativista no contexto da pandemia: desafios e contribuições}

Os princípios básicos dos cuidados paliativos vêm sendo cada vez mais discutidos nos cenários do cuidar, com desdobramentos em diversas situaçôes e grupos populacionais, desvelando sua potencialidade durante a crise da COVID-19. As estratégias direcionadas para apoiar pacientes e familiares contemplam princípios como aliviar os sintomas, garantir conforto no processo de morte, oferecer atendimento centrado no paciente, cuidar dos pacientes e seus familiares em relação aos aspectos físicos e espirituais e atuar com equipe multidisciplinar. ${ }^{(27,41,43)}$

Embora não se possam prever os desfechos do cuidado, os profissionais de saúde devem realizar o planejamento avançado de cuidados de acordo os fatores que são importantes para o quadro clínico do paciente. Além do controle adequado dos sintomas, que é um direito humano básico e fundamental para aliviar o sofrimento, independentemente 
das chances de sobrevivência, deve-se levar em consideração a espiritualidade, cuja importância é reconhecida no conforto e na promoção do bem-estar de pacientes e familiares. ${ }^{(27)}$

Os relatos de incertezas em relação à melhor intervenção a ser realizada, associados às angústias produzidas pela intensa carga de trabalho dos profissionais de saúde, não devem representar impedimentos para a aplicação dos princípios de cuidado paliativo no contexto da pandemia. Se a triagem for necessária para priorizar os cuidados, quando os leitos de terapia intensiva estiverem escassos, deve ser necessário comunicar isso de forma sensível e respeitosa aos pacientes e a suas famílias, sugerindo um caminho alternativo. ${ }^{(43)}$

Além da questão relacionada à infraestrutura dos serviços e às incertezas da doença, discutir e implementar os cuidados paliativos são pertinentes, nesse contexto de pandemia, pois as doenças crônicas aumentam o risco de complicaçôes pela COVID-19. Pacientes idosos e/ou com comorbidades tendem a evoluir para quadros de maior complexidade, principalmente com a síndrome do desconforto respiratório agudo. ${ }^{(23,28)}$

Com base nesse perfil de pacientes acometidos pela COVID-19, as medidas a serem aprimoradas e implementadas com maior vigor em unidades de saúde estavam relacionadas ao desconforto respiratório e ao manejo da dor (por meios farmacológicos e não farmacológicos). ${ }^{(20,27,31)}$ Pensar em cuidados paliativos no manejo da COVID-19 implica em refletir sobre a tomada de decisão baseada no estado clínico do paciente e na implementação de medidas para conforto.

Quando o paciente é o centro e seu conforto é o foco do cuidado, os princípios paliativistas são aplicados em sua essência, proporcionando um olhar holístico e centrado em um indivíduo, envolto por sentimentos de medo e incerteza. ${ }^{(23)}$ Dentre os cuidados relatados para o manejo da COVID-19 que se sobrepóem ao cuidado físico, destacam-se a disponibilidade de canais de comunicação entre familiares, proporcionando um momento de despedida e/ou esperança e a utilização de exercícios de respiração no controle da ansiedade de casos leves. ${ }^{(27,39,41,44)}$

Avaliar o quadro clínico e o perfil de pacientes com COVID-19 é determinante na tomada de de- cisão sobre a indicação de procedimentos invasivos e ressuscitação. Muitos preferem morrer em seu ambiente familiar, e não em uma unidade de cuidados intensivos. Nesses casos, o respeito às preferências do paciente e da família deve ser valor indispensável e pode ser identificado pelo consentimento informado da família. ${ }^{(24,25)} \mathrm{O}$ cuidado paliativo se faz necessário para proporcionar conforto, em oposição às medidas invasivas, que, muitas vezes, trazem sofrimento e poucos benefícios ao paciente e/ou familiar. $^{(19)}$

Como forma de mitigar os impactos diários da pandemia, os princípios da comunicação terapêutica devem ser utilizados para facilitar o diálogo com as famílias. Essa estratégia pode contribuir para minimizar o estresse, favorecendo a adaptação de novas rotinas familiares, impostas pelas mudanças bruscas, inclusive com a impossibilidade de vivenciar as práticas comuns de apoio social e cerimônias religiosas nos funerais. ${ }^{(16,28,39,45)}$

A instabilidade dos pacientes acometidos pela COVID-19, que, muitas vezes, apresentam piora rápida do quadro clínico, exigiu adaptação imediata dos serviços de saúde, para enfrentamento da pandemia e o gerenciamento do atendimento em saúde para essa demanda intensa e específica. ${ }^{(21,36)}$

A tensão diante do manejo da COVID-19 também evidenciou questôes de vulnerabilidade em saúde, equidade na assistência e igualdade de gênero e tratamento. Reforça-se a ação da equipe de cuidados paliativos em humanizar as práticas destinadas ao paciente e respeitar sua individualidade, assegurando que os indivíduos recebam cuidados de alta qualidade, com respeito à sua forma de ver e viver a vida. ${ }^{(34)}$

\section{Conclusão}

Os princípios dos cuidados paliativos contribuem para o cuidado integral, mas sua implementação no contexto de crise e pandemia é um desafio, devido ao desconhecimento dos profissionais de saúde, pacientes e familiares acerca desta abordagem. Os cuidados paliativos promovem uma visão holística do paciente e do cuidado, com respeito à vida e à 
dignidade humana. Esta abordagem contribui para o cuidado humanizado e respeitoso, acolhendo as preferências dos pacientes e familiares. É importante ampliar a formação dos profissionais de saúde para o cuidado paliativo, proporcionado condiçôes para aplicá-lo no cotidiano dos serviços de saúde, mesmo em cenários de crise e pandemia.

\section{Agradecimentos}

À Coordenação de Aperfeiçoamento de Pessoal de Nível Superior (CAPES; bolsa de pós doutorado para RSF e bolsa de doutorado para VRFC), ao Conselho Nacional de Ciência e Tecnologia (CNPq; bolsa de doutorado para LCS e bolsa de produtividade Pq1A para TMMM).

\section{Referências}

1. Kelvin AA, Halperin S. COVID-19 in children: the link in the transmission chain. Lancet Infect Dis. 2020;20(6):633-4.

2. Nathan N, Prevost B, Corvol H. Atypical presentation of COVID-19 in young infants. Lancet. 2020;395(10235):1481.

3. Goldman PS, van ljzendoorn MH, Sonuga-Barke EJ, Goldman PS, van ljzendoorn MH, Bakermans-Kranenburg MJ, et al.; Lancet Institutional Care Reform Commission Group. The implications of COVID-19 for the care of children living in residential institutions. Lancet Child Adolesc Health. 2020;4(6):e12.

4. World Health Organization (WHO). Definition of palliative care [Internet]. Genève: WHO; 2016[cited 2020 Aug 23]. Available from: https://www. who.int/news-room/fact-sheets/detail/palliative-care

5. World Health Organization (WHO). Clinical management of COVID-19 [Internet]. Genève: WHO; 2020[cited 2020 Aug 23]. Available from: https://www.who.int/publications/i/item/clinical-managementof-covid-19

6. Abdolrahimi $M$, Ghiyasvandian $S$, Zakerimoghadam M, Ebadi A. Therapeutic communication in nursing students: A Walker \& Avant concept analysis. Electron Physician. 2017;9(8):4968-77.

7. World Health Organization (WHO). Integrating palliative care and symptom relief into the response to humanitarian emergencies and crises: a WHO guide [Internet]. Genève: WHO; 2018[cited 2020 Aug 23]. Available from: https://apps.who.int/iris/ bitstream/handle/10665/274565/9789241514460-eng. pdf? sequence $=1$ \&isAllowed $=y$

8. Fadul N, Elsayem AF, Bruera E. Integration of palliative care into COVID-19 pandemic planning. BMJ Support Palliat Care. 2020;11(1):e002364.

9. Barbosa Filho VC, Tricco AC. Scoping review: a relevant methodological approach for knowledge synthesis in Brazil: a health literature. Rev Bras Ativ Fís Saúde. 2019;24(1):1-6.
10. Munn Z, Peters MD, Stern C, Tufanaru C, McArthur A, Aromataris E. Systematic review or scoping review? Guidance for authors when choosing between a systematic or scoping review approach. BMC Med Res Methodol. 2018;18(1):143.

11. Peters MD, Godfrey C, Mclnerney P, Soares BC, Khalil H, Parker D. Scoping reviews. In: Aromataris E, Munn Z, editors. Joanna Briggs Institute Reviewer's Manual. Australia: Joanna Briggs Institute; 2017.

12. Peters MD, Godfrey CM, Khalil H, Mclnerney P, Parker D, Soares CB. Guidance for conducting systematic scoping reviews. Int J Evid-Based Healthc. 2015;13(3):141-6.

13. Peters MD, Godfrey CM, Mclnerney P, Soares CB, Khalil H, Parker D. The Joanna Briggs Institute reviewers' manual 2015. Methodology for JBI scoping reviews [Internet]Australia: Joanna Briggs Institute; 2015. [ [cited 2020 Aug 23]. Available from https://nursing.Isuhsc.edu/JBI/ docs/ReviewersManuals/Scoping-.pdf

14. Tricco AC, Lillie E, Zarin W, O'Brien KK, Colquhoun H, Levac D, et al. PRISMA Extension for Scoping Reviews (PRISMA-ScR): checklist and Explanation. Ann Intern Med. 2018;169(7):467-73.

15. Moher D, Liberati A, Tetzlaff J, Altman DG; PRISMA Group. Preferred reporting items for systematic reviews and meta-analyses: the PRISMA statement. PLoS Med. 2009;6(7):e1000097.

16. Weaver MS, Wiener L. Wiener I. Applying palliative care principles to communicate with children about COVID-19. J Pain Symptom Manage. 2020;60(1):e8-11.

17. Calton B, Abedini N, Fratkin M. Telemedicine in the time of coronavirus. J Pain Symptom Manage. 2020;60(1):e12-4.

18. Powell VD, Silveira MJ. What should palliative care's response be to the COVID-19 epidemic? J Pain Symptom Manage. 2020;60(1):e1-3.

19. Hendin A, La Rivière CG, Williscroft DM, O'Connor E, Hughes J, Fischer LM. End-of-life care in the emergency department for the patient imminently dying of a highly transmissible acute respiratory infection (such as COVID-19). CJEM. 2020;22(4):414-7.

20. Nehls W, Delis S, Haberland B, Maier B0, Sänger K, Tessmer G, et al. Recommendations for treatment of patients with COVID-19 from the palliative care perspective V2.0 [Internet]. Germany: DGP; 2020 [cited 2020 Aug 23];(2):1-10. Available from: https://www.dgpalliativmedizin. de/images/DGP_Handlungsempfehlung_palliative_Therapie_bei_ COVID18_V2.0_English_version.pdf

21. Fausto J, Hirano L, Lam D, Mehta A, Mills B, Owens D, et al. Creating a palliative care inpatient response plan for COVID-19-the UW medicine experience. J Pain Symptom Manage. 2020;60(1):e21-6.

22. Ferguson L, Barham D. Palliative Care Pandemic Pack: A Specialist Palliative Care Service Response to Planning the COVID-19 Pandemic. J Pain Symptom Manage. 2020;60(1):e18-20.

23. Borasio GD, Gamondi C, Obrist M, Jox R; For the Covid-Task Force of Palliative Ch. COVID-19: decision making and palliative care. Swiss Med Wkly. 2020;150:w20233.

24. Rolanda K, Markusb M. COVID-19 pandemic: palliative care for elderly and frail patients at home and in residential and nursing homes. Swiss Med Wkly. 2020;24(150):e20235.

25. Curtis JR, Kross EK, Stapleton RD. The importance of addressing advance care planning and decisions about do-not-resuscitate orders during novel coronavirus 2019 (COVID-19). JAMA. 2020;323(18):1771-2.

26. Palliative care and the COVID-19 pandemic. Lancet. 2020;395(10231):1168. 
27. Bajwah S, Wilcock A, Towers R, Costantini M, Bausewein C, Simon ST, et al. Managing the supportive care needs of those affected by COVID-19. Eur Respir J. 2020;55(4):2000815.

28. Salako 0, Okunade K, Allsop M, Habeebu M, Toye M, Oluyede $\mathrm{G}$, et al. Upheaval in cancer care during the COVID-19 outbreak. Ecancermedicalscience. 2020;14(1):ed97.

29. Fan J. China's struggles with hospice care. The New Yorker [Internet]. 2020 [cited 2020 Aug 23]. Available from: https://www.newyorker. com/magazine/2020/04/06/chinas-struggles-with-hospice-care

30. Etkind SN, Bone AE, Lovell N, Cripps RL, Harding R, Higginson IJ, et al. The role and response of palliative care and hospice services in epidemics and pandemics: a rapid review to inform practice during the COVID-19 pandemic. J Pain Symptom Manage. 2020 ;60(1):e31-40.

31. Fusi-Schmidhauser T, Preston NJ, Keller N, Gamondi C. Conservative management of COVID-19 patients-emergency palliative care in action. J Pain Symptom Manage. 2020;60(1):e27-30.

32. Tran DL, Lai SR, Salah RY, Wong AY, Bryon JN, McKenna MC, et al. Rapid de-escalation and triaging patients in community-based palliative care. J Pain Symptom Manage. 2020;60(1):e45-7.

33. Lovell N, Maddocks M, Etkind SN, Taylor K, Carey I, Vora V, et al. Characteristics, symptom management, and outcomes of 101 patients with COVID-19 referred for hospital palliative care. J Pain Symptom Manage. 2020;60(1):e77-81.

34. Rosa WE, Shook A, Acquaviva KD. LGBTQ+ inclusive palliative care in the context of COVID-19: Pragmatic recommendations for clinicians. J Pain Symptom Manage. 2020;60(2):e44-7.

35. Humphreys J, Schoenherr L, Elia G, Saks NT, Brown C, Barbour S, et al. Rapid implementation of inpatient telepalliative medicine consultations during COVID-19 pandemic. J Pain Symptom Manage. 2020;60(1):e54-9.

36. Costantini M, Sleeman KE, Peruselli C, Higginson IJ. Response and role of palliative care during the COVID-19 pandemic: A national telephone survey of hospices in Italy. Palliat Med. 2020;34(7):889-95.

37. Davies A, Hayes J. Palliative care in the context of a pandemic: similar but different. Clin Med (Lond). 2020;20(3):274-7.

38. Sun H, Lee J, Meyer BJ, Myers EL, Nishikawa MS, Tischler JL, et al. Characteristics and Palliative Care Needs of COVID-19 Patients Receiving Comfort-Directed Care. J Am Geriatr Soc. 2020;68(6):1162-4.

39. Romanò M. [Between intensive care and palliative care at the time of CoViD-19]. Recenti Prog Med. 2020;111(4):223-30. Italian.

40. Radbruch L, Knaul FM, de Lima L, de Joncheere C, Bhadelia A. The key role of palliative care in response to the COVID-19 tsunami of suffering. Lancet. 2020;395(10235):1467-9.

41. Feder SL, Akgün KM, Schulman-Green D. Palliative care strategies offer guidance to clinicians and comfort for COVID-19 patient and families. Heart Lung. 2020;49(3):227-8.

42. Chidiac C, Feuer D, Naismith J, Flatley M, Preston N. Emergency palliative care planning and support in a COVID-19 pandemic. J Palliat Med. 2020;23(6):752-3.

43. Peate I. COVID-19 and palliative care. Br J Nurs. 2020;29(8):455.

44. Arya A, Buchman S, Gagnon B, Downar J. Pandemic palliative care: beyond ventilators and saving lives. CMAJ. 2020;192(15):E400-4.

45. Wallace CL, Wladkowski SP, Gibson A, White P. Grief during the COVID-19 pandemic:considerations for palliative care providers. J Pain Symptom Manage. 2020;60(1):e70-6. 